“La complejidad de los problemas actuales no se puede resolver con el mismo nivel de pensamiento que los generó... Nuestra forma de pensar tradicional nos tiene aprisionados en esquemas que explican nuestra incapacidad de encontrar nuevos caminos"

ALBERT EI NSTEI N

\title{
EL PAPEL ACTUAL DE LA ACADEMIA COMO ACTOR FUMDAMEMTAL DEL DESARROLLO LOCAL Y REGIOMAL
}

\author{
Fecha de Recepción: Diciembre 10 de 2006 \\ Fecha de Aceptación: Enero 15 de 2007
}

Marco Antonio Ramírez Ospina*

\section{SÍ NTESIS}

El fortalecimiento del proceso investigativo en la Universidad La Gran Colombia, ha permitido identificar las necesidades actuales de los municipios del Quindío en materia de ordenamiento territorial, ratificando la pertinencia de la participación de la academia en los procesos de desarrollo. Es por eso que a través de propuestas concretas, la Facultad de Arquitectura se vislumbra como protagonista de estos procesos, dentro de su compromiso esencial con un Desarrollo Regional fundamentado en la sinergia entre actores locales.

\section{Palabras clave}

Desarrollo, Academia, Gestión, Ordenamiento Territorial, Arquitectura.

\section{ABSTRACT}

The investigation enforcement process in La Gran Colombia University, has permitted to identify nowadays necessities in the towns of Quindío about territorial planning, verifying the pertinence of the academy in the development process. Due to this through concrete propositions, the Architecture Faculty is positioned itself as the essential staring of these processes, according with its compromise with Regional Development settled in the synergy between local actors.

\section{Key words}

Development, Academy, Management, Territorial Planning, Architecture.

* Arquitecto, Especialista en Derecho Urbano, Coordinador Investigaciones Facultad de Arquitectura Universidad La Gran Colombia Armenia 


\section{CONTEXTO (SENTIR):}

La visión paternalista generalizada que caracteriza la sociedad latinoamericana, ha llevado a considerar tradicionalmente al Estado como el único actor del desarrollo territorial, social y económico de los pueblos. El concepto mismo de "Estado" ha sido mal manejado culturalmente, pues tiende a confundirse con el de "Gobierno", entidad institucional designada por el pueblo para adoptar decisiones y administrar al país en cada uno de sus niveles, excluyendo radicalmente al grueso de sus integrantes representados por la comunidad en general, por todos y cada uno de los ciudadanos e instituciones que conforman la Nación. De este modo, y tal vez asumiendo posiciones cómodas, se ha culpado al "Estado" de todas las enfermedades y dolencias de la patria, sin tener en cuenta que el "Estado" somos todos, representados por quienes nosotros mismos hemos designado para tal fin. El enfoque actual apunta a consolidar el concepto de "EstadoNación"(KARL HAUSHOFER) y a difundirlo entre el grueso de los ciudadanos, en busca de fortalecer los procesos democráticos participativos de nuestros países.

Igualmente, para nadie es un secreto que en el contexto local, la institucionalidad académica se ha mantenido exenta de los procesos reales de intervención sobre el territorio, quedando marginada de las apuestas para el desarrollo. Este aislamiento, sumado con otros factores, ha llevado a un detrimento paulatino de la capacidad operativa de los entes gubernamentales para planear integralmente el desarrollo de sus territorios, para formular y ejecutar proyectos que le apunten directamente a elevar los índices de Cali- dad de Vida de sus habitantes, generando de este modo desesperanza, baja credibilidad, ausencia de legitimidad en sus procesos y por ende, dejando serios vacíos en la gobernancia pública.

Bajo nociones tradicionales, el Desarrollo se consideraba como algo cuantificable, cuyo sustrato era la acumulación, la inversión y la formación de capacidad productiva. Ahora, bajo las visiones renovadoras y actuales, que han dado respuesta a las aceleradas dinámicas de la ciudad y de su entorno, el verdadero Desarrollo es principalmente un "proceso de activación y canalización de fuerzas sociales, de mejoría en la capacidad asociativa, de ejercicio de la iniciativa y de la inventiva" (FURTADO, 1989). Se trata entonces de un proceso social y cultural, y sólo secundariamente económico. El desarrollo se produce cuando en la sociedad se manifiesta una energía capaz de canalizar, de forma convergente, fuerzas que estaban latentes o dispersas.

Teniendo en cuenta estos factores, se observa cómo este concepto se debe construir colectivamente definiendo la "Gestión del Desarrollo" como el proceso que está en la capacidad de convocar a los actores y las instituciones para orientar los procedimientos de la gestión pública y privada, aprovechar los recursos materiales, humanos, cognitivos y psico-sociales. Ahora bien, ien cabeza de quien está la Gestión del Desarrollo?

El actor local es aquel cuya acción contribuye a desplegar las potencialidades existentes en la sociedad. También incorpora a quienes obstaculizan, a través de diversas modalidades de acción u omisión, las posibilidades de desarrollo. 
La academia en su misión constante de conservar, difundir, adaptar y generar conocimiento, cultura y tecnología aplicados a las realidades del contexto, se debe considerar como un actor local fundamental para el DesarroIlo. Debe proyectarse como esa fuerza latente, capaz de hacer converger procesos sociales y culturales, estimulando en su comunidad académica la investigación, el liderazgo y la integración armónica del hombre con su entorno. La Universidad, como espacio de construcción social, está en la obligación de fomentar el diálogo de los valores y los saberes de los nuevos avances científicos y sociales del mundo a fin de consolidar la identidad y la integración regional y nacional.

Asumiendo estos compromisos, consignados en la Misión Institucional, la Facultad de Arquitectura de la Universidad La Gran Colombia, por intermedio de su "Laboratorio de Arquitectura y Hábitat Urbano Regional" (LAHUR), participa activamente en los procesos de transformación y construcción de ciudades soportada en investigación aplicada que genera proyectos urbanos y arquitectónicos, formulando estrategias a partir de problemáticas específicas o de potencialidades del territorio, que promuevan en su producción y desarrollo, espacios de reflexión y discusión en torno a las dinámicas territoriales. Este esfuerzo por saber cómo estamos, en tiempo y espacios reales, proporciona herramientas políticas para incentivar la inversión y el desarrollo empresarial, dinamizar una planeación estratégica y participativa más acertada, generar políticas de vivienda, empleo y desarrollo humano acordes con el contexto regional, así como la capacidad de anticiparnos a eventos de escala global que podrían afectarnos di- rectamente (Metropolización, Regionalización, Globalización, Internacionalización, Tratados de Libre Comercio, etc.).

EI LAHUR, en sus Líneas de Investigación, tiene como propósito abrir un espacio de estudio en el ámbito de lo territorial y lo proyectual; una reflexión que permita aproximarse al análisis de los procesos de crecimiento generados a partir de las relaciones entre la ciudad y el territorio desde el desarrollo histórico de los poblados hasta la conurbación metropolitana, convirtiendo así, la reflexión sobre la ciudad, en proyectos arquitectónicos que permitan verificar las ideas y discusiones que desde la academia se generan para el mejoramiento de la calidad de vida urbana de los municipios del Quindío.

Por tales motivos, asumiendo el rol protagónico que le corresponde a la academia como actor fundamental del desarrollo, se ha definido como estrategia de acción generar escenarios de interacción en los cuales los proyectos, programas y productos de la facultad de Arquitectura sean presentados de manera directa a la comunidad. Ejemplo de ello fue la actividad denominada “1er FORUM ACADÉMI CO-MONTENEGRO 2005- Una nueva convivencia a través del desarrollo integral de la comunidad". Este espacio permitió hacer presencia institucional en el Municipio de Montenegro e impactar positivamente su desarrollo local, generando un acercamiento directo entre la comunidad académica y los ciudadanos, al tiempo que se exhibieron los proyectos integradores de Taller diseñados para el contexto específico del Municipio y se realizaron actividades lúdicas para sus habitantes. 


\section{REALI DADES Y PLANEACI ÓN PARA EL DESARROLLO (PENSAR):}

En busca de lineamientos para la construcción de un Ordenamiento Territorial para el desarrollo humano sostenible, el LAHUR circunscribe a los municipios del Quindío en ciertas realidades de las cuales hacen parte, en aras de proyectar sus escenarios tendenciales de desarrollo:

La estrategia de actuación de LAHUR de la Facultad de Arquitectura con los municipios, hace parte de una visión conjunta que plantea una Integración para el desarrollo regional integral, consolidando el concepto de Ciudad Región con especialización funcional e interdependencia de sus núcleos urbanos, a través de áreas de interacción, no necesariamente geográficas o territoriales, que permitan generar corredores de competitividad, aprovechando la posición estratégica del municipio en el escenario departamental y en las dinámicas sociales del EJE CAFETERO, entre las que se encuentra el turismo como actividad destacada. De este modo, basándose en el patrimonio natural, y arquitectónico existente en el municipio, en su biodiversidad y en los aceptables niveles de infraestructura dejados por el proceso de reconstrucción, se podrán fortalecer las vocaciones económicas planteadas en sus diferentes instrumentos de planificación, como lo son el fortalecimiento tanto del sector agrícola como de la Agroindustria, la potencialización del Turismo y sobretodo la prestación de bienes y servicios atendiendo al proceso de "Terciarización de la economía" al cual estamos avocados regional e internacionalmente.
Realizando una mirada regional, se plantean diversos mega proyectos estratégicos que contrastan con procesos incipientes de planeación en la escala local de la mayoría de los municipios, y consecuentes niveles deficitarios en el seguimiento de políticas e implementación de estrategias que permitan ejecutar lo planteado en los procesos de planeación territorial y socio económica. Dada la baja capacidad operativa de los departamentos administrativos de planeación y las innegables dificultades financieras de inversión, las administraciones municipales han tendido a priorizar el desarrollo social y económico satisfaciendo las necesidades del día a día de la población, pero paradójicamente, tales esfuerzos no han podido aumentar los bajos índices de desarrollo humano evidentes en la región que por el contrario van cada vez más en caída, como consecuencia de los indeterminados problemas demográficos (por aumento en unos y disminución de población en otros) que generan incremento en la demanda de servicios sociales y crecimiento desbordado de las cabeceras urbanas que se perciben cada vez más dispersas, conflictivas y marginales.

Es por eso que se hace necesario y perentorio realizar una exploración de alternativas económicas ${ }^{1}$, diversificando los renglones productivos de los municipios y generando de este modo modelos de desarrollo territorial asociativos que permitan convertir las abundantes ventajas comparativas verdaderas ventajas competitivas que proyecten a la región en el escenario interna-

1 Las directrices socio económicas planteadas en este punto corresponden en su mayoría a la propuesta del Consultor- Investigador José I gnacio Rojas Sepúlveda. En su ponencia DESARROLLO LOCAL Y ACTORES SOCIALES EN EL QUINDÍO 19902004. 
cional. La planificación del desarrollo se debe orientar por el peso de las ventajas comparativas existentes como posición geoestratégica, bondades de configuración geográfica y paisajística, adecuada infraestructura vial y de servicios, entre otras. Frente a este panorama se presenta paradójicamente una escasa reflexión sobre las variables que generan competitividad:

- Cualificación estratégica del talento humano.

- Procesos de investigación y desarrollos tecnológicos.

- Nueva visión institucional

- Generación de valor agregado a procesos productivos locales.

- Especialización de sub-sectores económicos.

- Consolidación de cadenas productivas.

- Disfuncionalidad entre la concepción del desarrollo y sus prácticas cotidianas.

- Aplicación de instrumentos de gestión y financiación del suelo.

La carencia de un sólido proceso de desarrollo se explica por una ruptura en la concepción "del desarrollo". Los proyectos de planificación no van mas allá de plantearse como una construcción de imaginarios locales frente al desarrollo de voluntades de actores políticos decisores que generalmente satisfacen intereses particulares. Mientras tanto, las reales prácticas del proceso de planificación se expresan en:

- Diversos ejercicios aislados de carácter sectorial-institucional.

- Programas de ejecución de proyectos de bajo impacto en los que no se traducen las intencionalidades de la planeación.

- Los esfuerzos por un desarrollo in tegral no se ven favorecidos a consecuencia de la desarticulación de los actores públicos, privados y comunitarios.

Las acciones para el desarrollo socio- económico y territorial presentan multiplicidad de actuaciones sectoriales. Diferentes agentes locales abordan temáticas sectoriales sin que medie entre ellos una articulación y construcción colectiva. Se evidencia igualmente en esta sociedad una baja capacidad de flujos de información, de acuerdos sectoriales, de cooperación y de asociatividad sostenible de los agentes institucionales que han generado un inminente deterioro del concepto de lo público traducido en bajos niveles de cultura ciudadana, una sociedad civil que discute y propone, pero no actúa, ciudadanos con baja noción de su incidencia en las decisiones sobre asuntos colectivos, una clase dirigente local y unos gobiernos con baja noción, promoción y defensa de los intereses generales, una evidente ruptura del sector oficial y los sectores privado, académico y comunitario, ausencia de control social ciudadano, que llevan irremediablemente a una débil planificación de largo plazo y participativa sobre lo público.

Por las razones anteriormente expuestas, la asociatividad se convierte en un factor clave en el éxito competitivo del territorio, planteada igualitariamente entre el sector público y el privado para generar proyectos políticos y agendas compartidas; entre el sector productivo, el sector investigativo y el sector gobierno, para el desarrollo de sistemas de aprendizaje y de innovación; entre la academia el sector público y las ONG's, para acompañar procesos de planificación y ejecutando proyectos de desarrollo; entre empresas, originando 
clusters productivos; y finalmente entre territorios dando origen a regiones asociativas de planificación que permitan gestionar integralmente el escenario urbano regional.

\section{PROPUESTA DE I NTERVENCI ÓN (HACER):}

Revisadas las necesidades actuales de los municipios del departamento en materia de ordenamiento territorial, se ratifica la pertinencia de la participación de la academia en los procesos de desarrollo municipal y regional. Es por eso que a través de propuestas concretas, la Facultad de Arquitectura se vislumbra como protagonista esencial de estos procesos.

Dentro de este contexto, se presento esta propuesta específica para el municipio de Montenegro, en la cual se realizó un diagnóstico completo del Plan Básico de Ordenamiento Territorial y del Plan de desarrollo Municipal vigentes, identificando sus propuestas estructurantes y puntos de articulación, bajo el marco de la Ley de Desarrollo Territorial $^{2}$ y de sus decretos reglamentarios, en aras de encuadrar una propuesta de apoyo técnico por parte de la Facultad hacia el municipio, en la cual se presentaron proyectos concretos de intervención y de generación de normativa, dentro de los convenios establecidos entre la Universidad y la entidad territorial.

La Facultad de Arquitectura desarrolló durante los pasados semestres académicos un trabajo de bloque en el municipio de Montenegro, en el cual, puso las 385 personas que la integran a pensar, proponer y desarrollar soluciones concretas a las necesidades ac-

2 Ley 388 de 1997 tuales del municipio. Esta fuerza de trabajo se desplegó a través de tres escenarios: El primero de ellos son las aulas, en las cuales se adelantaron ejercicios académicos en los diez semestres para el contexto específico de Montenegro, de los cuales se extrajo como producto la realización de diversos trabajos de grado, teóricos y proyectuales para este contexto; el segundo escenario es el LAHUR, que funciona a manera de oficina de proyectos, integrado por estudiantes de últimos semestres en ejercicio de su pasantía, profesionales egresados de la facultad, asesores y el cuerpo docente, conformado por un grupo interdisciplinario de profesionales que están al frente de los proyectos propuestos; el tercer escenario es la Embajada Gran Colombiana, que tiene asiento físico en el municipio y ha sido la encargada de servir de canal de comunicación entre la comunidad y la Facultad de Arquitectura mediante un trabajo de planeación participativa.

La lectura de los insumos de planeación municipal se realizó de manera sistemática, analizando paralelamente ambos documentos (P.B.O.T. y Plan de Desarrollo) a través de atributos y dimensiones. Se obtuvo en primer lugar un documento que extrae los aspectos más importantes del Plan de Desarrollo en materia de proyecto urbano, encontrando que la estrategia de "Obras Públicas con Participación Ciudadana" encuadraba perfectamente con el marco de trabajo institucional. El segundo producto arrojado es una matriz de categorías que recoge las propuestas específicas del P.B.O.T. en las cuales la Facultad pudiese tener participación, clasificándolas según el capitulo, la estrategia, el programa los subprogramas, las líneas de acción y los proyectos puntuales. 
Dada la vaguedad observada en la formulación de políticas del PBOT y la ausencia de proyectos puntuales, presentes en diversos planes de ordenamiento de todo el país, de acuerdo el estudio y las reuniones realizadas con el equipo de la administración, se encontraron como necesidades prioritarias del municipio los siguientes proyectos, en los cuales la Facultad de Arquitectura puso a disposición la fuerza de trabajo del laboratorio para ser desarrollados a través de consultoría:

\section{1- Proyecto de modelo de ocupación} para las zonas de Expansión Urbanas proyectadas, equivalentes a 93ha o $5961 \mathrm{~m} 2$ dirigidas a suplir la demanda actual y futura de VIS. El Proyecto parte de un inventario de los bienes inmuebles de propiedad del ente territorial y un estudio de titularidad de inmuebles particulares potenciales de ser desarrollados, apuntando a la implementación del banco de tierras para dotar al municipio, a través de este instrumento, de los espacios físicos necesarios para emplazar colectiva o aisladamente, la construcción de VIS dirigida a los reductos poblacionales mas vulnerables del municipio.

\section{2- Proyecto integral de Espacio Pú-} blico Urbano. Esta propuesta apunta a realizar una intervención física de rehabilitación del centro que permita ofertar espacios urbanos de interés turístico, en los cuales la comunidad misma, se vea beneficiada económicamente de las dinámicas generadas por los recorridos de la población flotante que llega al municipio. La propuesta consiste en la elaboración del Manual de Espacio público de Montenegro que contempla la ampliación de andenes y de espa- cios peatonales alrededor del centro, articulando la plaza principal con una serie de recorridos temáticos urbanos entorno a los bienes de interés cultural existentes, generando tensiones hacia unos posibles miradores turísticos sobre la cuenca del río Roble y hacia unos puntos de información y servicio ubicados en los accesos principales al casco urbano.

3- Rehabilitación del corredor vial suburbano Montenegro-PuebloTapao y potencialización de este eje como epicentro de la actividad turística del municipio. Esta propuesta se plantea como un macroproyecto de ampliación y recuperación física, económica y ambiental de este carreteable como espacio público rural, en el cual se propondrían los esquemas de negociación adecuados para adquirir unas fajas de terreno paralelas a la vía, con el objeto de realizar un paseo peatonal, con ciclo rutas, señalización y arborización que integre el corregimiento de PuebloTapao y sus diversas dinámicas con el centro del municipio de Montenegro, pasando por puntos de tensión importantes como lo son el Parque del Café y el mirador de Canes para fortalecer y formalizar las actividades económicas derivadas de la actividad turística y mitigar los impactos ambientales que actualmente se generan.

4- Realización de módulos de Vivienda de I nterés Social, que permita suplir las demandas actuales y futuras del municipio y regularizar o reubicar los asentamientos humanos irregulares existentes desde dos frentes, dirigidos a la demanda actual y futura de VIS para las áreas de desarrollo prioritario a través del 
esquema de autoconstrucción. La propuesta partiría del diseño arquitectónico de diferentes módulos de vivienda, aprovechando las potencialidades ambientales del municipio y llegaría a proponer el diseño urbano de los emplazamientos de acuerdo a los entornos específicos.

\section{5- Proyecto de rehabilitación y cons- trucción de infraestructuras co-} munitarias, que busque dotar a los diferentes barrios de espacios físicos para la reunión, recreación, esparcimiento y práctica del deporte, consistente en adecuación de casetas comunales, polideportivos, parques y espacios verdes para el sano disfrute del tiempo libre y la integración social a través del esquema de participación comunitaria y autogestión.

Cada una de estas propuestas, ha sido abordada como tema de estudio en un Taller de Proyectación en la Facultad, apuntando a la consolidación de un macroproyecto urbano que permita impactar de manera importante en la comunidad a través de las estrategias planteadas por cada uno de los proyectos puntuales, en concordancia con la política de interacción social entre la administración y las comunidades, trascendiendo mas allá de los límites de los periodos de un gobierno. Dicho macroproyecto se concibe de manera global, pero podría estar presto a desarrollarse por etapas de acuerdo a cada una de las estrategias planteadas, apuntando a generar un verdadero desarrollo sostenible a escala humana para el Municipio de Montenegro y en especial para sus habitantes, como modelo de intervención para otras municipalidades de la región.
La implementación de este macroproyecto, se viabiliza aplicando las políticas consignadas en el PBOT del Municipio. Sin embargo, en materia de normatividad urbanística los Municipios del Quindío presentan en su mayoría un panorama incierto. Los componentes de formulación de sus planes de ordenamiento no definen políticas claras para el desarrollo territorial, siendo éstos tan solo documentos de carácter teórico que en su mayoría se limitan a reseñar, de manera general, lo establecido en la ley 388, con carencia de políticas concretas de intervención y que en escasas ocasiones proponen proyectos específicos de actuación que detonen desarrollo. Se evidencian también una serie de vacíos que se hace necesario entrar a suplir de manera inmediata, como lo son la identificación de Planes Parciales y Unidades de Actuación Urbanística, así como la implementación de Instrumentos de Gestión y Financiación puesto que muchos Planes de Ordenamiento no los dejaron establecidos.

Las necesidades actuales que presentan estos municipios, hacen evidente la ausencia de políticas claras de desarrollo territorial a escala regional y local, razón por la cual se hace urgente la formulación de normatividad urbanística específica que establezca un ordenamiento territorial claro, bajo el cual la sociedad pueda establecer cuál es el camino de su desarrollo, a través de la aplicación de los diferentes instrumentos de gestión y de repartición equitativa de cargas y beneficios. Ante esta realidad se presentan dos alternativas de actuación:

1- Realizar por iniciativa de los municipios y acatando el mandato de la ley, los códigos urbanísticos que contengan las fichas normativas y 
la definición de los planes parciales necesarios para el ordenamiento del territorio.

2- Dar cumplimiento a lo establecido en la ley 902 de 2004 y en el Dec. 4002 de 2004 reglamentario de la ley 388 que en su Artículo $5^{\circ}$ plantea la Revisión de los planes de ordenamiento territorial: "Los Concejos municipales o distritales, por iniciativa del alcalde y en el comienzo del período constitucional de este, podrán revisar y ajustar los contenidos de largo, mediano o corto plazo de los Planes de Ordenamiento Territorial, siempre y cuando haya vencido el término de vigencia de cada uno de ellos, según lo establecido en dichos planes".

Dado que en la mayoría de los Municipios, el Plan de Ordenamiento no ha sido revisado aún, ni en sus normas generales ni en las complementarias y que la vigencia de éstos se extiende aún por otro periodo de gobierno, se hace viable realizar una revisión ordinaria de estos documentos en sus normas generales, teniendo en cuenta que a partir del primero de enero del 2008 se encontrarán en curso los periodos de ejecución del largo plazo. Tales revisiones, permitirán realizar cambios significativos en las políticas territoriales de los municipios, articulándolas con lo establecido en las propuestas de los nuevos planes de desarrollo, además que abrirían la posibilidad de formular una normatividad urbanística coherente, ajustada a los requerimientos legales actuales, que desarrolle instrumentos de gestión y financiación del suelo a través de los cuales el estado involucre a los actores privados en el desarrollo del territorio y por ende de las comunidades.
La intervención de la academia se hace pertinente, dado que para poder emprender estos procesos de revisión de las políticas de Ordenamiento se hace necesaria la realización, en primera instancia, del Expediente Municipal (Art. 112 Ley 388), instrumento de carácter obligatorio para poder dar viabilidad a la revisión de los planes de ordenamiento en todos los municipios del país, trabajo que no ha sido adelantado aún por la mayoría de ellos para la vigencia actual.

Finalmente, se plantea la intervención de la academia en los contextos regionales a través del montaje e implementación del Observatorio del Desarrollo, en conjunto con las entidades del orden departamental y municipales y articulado con la red mundial de observatorios de la agencia Hábitat-NU, escenario que se crea con el objeto de llenar el vacío dejado por los bajos niveles de gobernancia y por la ausencia de indicadores urbanos, ambientales económicos, sociales y territoriales, que permitan evaluar los avances en materia de ordenamiento y planificación, el cual espera constituirse como un actor local fundamental para los procesos de desarrollo regional, aportando estudios completos que diagnostiquen las necesidades reales de las poblaciones desde una visión investigativa y técnica, apuntando a cumplir los Objetivos del Milenio planteados por el pacto Hábitat II y suscritas por el estado Colombiano. El potencial institucional que posee la Universidad, está siendo aprovechado para realizar propuestas que vayan más allá de la intervención puntual, y que se enfoquen en realizar diagnósticos globales y establecer directrices que permitan generar modelos de ocupación sostenibles a través de los proyectos que se desarrollen en nuestros municipios, desde los diferentes campos profesio- 
nales que se pueden ofertar. Es por eso que se aprovechará la capacidad de gestión que posee la Universidad, en asocio con sus aliados estratégicos ${ }^{3}$, para aportar de manera concreta al desarrollo de nuestros municipios, desarrollando intervenciones que enaltezcan la imagen de la institución y que permitan reconocerla como un actor fundamental en el progreso de nuestras ciudades y en general de nuestra región.

Teniendo en cuenta la política social y comunitaria que se ha venido desarrollando con éxito desde las administraciones municipales, la Universidad ha potencializado al máximo un trabajo de planeación participativa, en el cual los líderes comunitarios juegan un papel fundamental en el montaje y ejecución de los proyectos, en interacción constante con nuestros docentes y estudiantes, asumiendo frontalmente las responsabilidades que posee la academia como detonante y actor fundamental en el desarrollo de un territorio.

“Una adecuada propuesta de gobierno debe estar fundamentada en la construcción de una ciudad con equidad, en la que el estado cumpla ese papel para la consecución del equilibrio social, brindándole a todas y todos los ciudadanos las mismas condiciones sociales para que puedan desarrollar libremente su proyecto de vida y ser con ello un actor social..."

\section{Sergio Fajardo Valderrama \\ Alcalde de Medellín}

3 Alcaldías, Gobernación del Quindío, Corporación Autónoma Regional, redes universitarias, entre otras.

\section{BI BLI OGRAFÍ A}

DESARROLLO LOCAL Y ACTORES SOCI ALES EN EL QUINDÍO 1990-2004 J osé I gnacio Rojas Sepúlveda. Consultor- Investigador.

Condiciones mínimas de calidad Facultad de Arquitectura U.G.C. Armenia 2005.

PROYECTO EDUCATIVO INSTITUCIONALUniversidad la Gran Colombia, Armenia, 2002.

\section{PLAN BÁSICO DE ORDENAMIENTO TERRITORI AL DE MONTENEGRO.}

PLAN DE DESARROLLO MUNICIPAL 20042007: Montenegro de Todos.

AGENDA INTERNA DE PRODUCTIVIDAD Y COMPETITIVIDAD DEL QUI NDÍ O-Gobernación del Quindío, Cámara de Comercio de Armenia, Departamento Nacional de Planeación, 2005.

Proyecto de investigación Observatorio urbano regional del Quindío - Marco Antonio Ramirez- Docente investigador FAUGC. 